\title{
On the Frame Properties of System of Exponents with Piecewise Continuous Phase
}

\author{
Saeed Mohammadali Farahani ${ }^{1}$, Tofig Isa Najafov ${ }^{2}$ \\ ${ }^{1}$ Institute of Mathematics and Mechanics of NASA, Baku, Azerbaijan \\ ${ }^{2}$ Nakhchivan State University, Nakhchivan, Azerbaijan \\ Email: saeedzfarahani@gmail.com, tofiq-necefov@mail.ru
}

Received January 14, 2013; revised April 3, 2013; accepted April 10, 2013

Copyright (C) 2013 Saeed Mohammadali Farahani, Tofig Isa Najafov. This is an open access article distributed under the Creative Commons Attribution License, which permits unrestricted use, distribution, and reproduction in any medium, provided the original work is properly cited.

\begin{abstract}
A double system of exponents with piecewise continuous complex-valued coefficients are considered. Under definite conditions on the coefficients the frame property of this system in Lebesgue spaces of functions is investigated. Such systems arise in the spectral problems for discontinuous differential operators.
\end{abstract}

Keywords: System of Exponents; Frame Property; Perturbation

\section{Introduction}

Consider the following system of exponents

$$
\left\{\mathrm{e}^{\mathrm{i} \lambda_{n} t}\right\}_{n \in Z},
$$

where $\left\{\lambda_{n}\right\} \subset C$ is a sequence of complex numbers, $Z$ are integers. Systems (1) are model ones while studying spectral properties of differential operators. Under suitable choice of the bounded variation function $\sigma(t)$ on the segment $[-a, a]$ they are eigenfunctions of first order differential operator $D u=\frac{\mathrm{d} u}{\mathrm{~d} t}$ with an integral condition of the form $\int_{-a}^{a} u(t) \mathrm{d} \sigma(t)=0$.

For this reason, many mathematicians appealed to study of basis properties of the systems form (1) in different spaces of functions. If the operator $D$ is considered in the Lebesgue space $L_{p}(-a, a), 1 \leq p<+\infty$, then its natural domain of definition is the Sobolev space $W_{p}^{1}(-a, a)$, i.e. the space consisting of absolutely continuous on $[-a, a]$ functions, whose derivatives belong to $L_{p}(-a, a)$ and the relation

$$
D u=\frac{\mathrm{d} u}{\mathrm{~d} t}=\lambda u(t)
$$

holds a.e. on all the segment $[-a, a]$.

Apparently, the first results for basis properties of the systems of the form (1) in the spaces $L_{p}, 1 \leq p \leq+\infty$, $\left(L_{\infty} \equiv C[-a, a]\right)$ belong to the famous mathematicians
Paley P.-N. Wiener [1] and N. Levinson [2]. In sequel, this direction was developed in the investigations of many mathematicians. For more detailed information see the monographs of R. Young [3], A. M. Sedletskii [4], Ch. Heil [5], O. Christensen [6] (and also the papers [79]) and their references. There is also the survey paper [10].

Many problems of mechanics and mathematical physics reduce to discontinuous differential operators, i.e. to the case when the domain of definition of a differential operator is not connected. It should be noted that the systems of the form

$$
\left\{\mathrm{e}^{\mathrm{i} \lambda_{n}(t)}\right\}_{n \in Z}
$$

where $\lambda_{n}(t)$ has the representation

$$
\lambda_{n}(t)=n t-\alpha(t) \operatorname{sign} n+\beta_{n}(t), \text { as } n \rightarrow \infty .
$$

arise as eigen functions of appropriate differential operators while solving many problems of mechanics and mathematical physics by the method of separation of variables. The following system is a trivial example of the case under consideration

$$
S_{n}(t)= \begin{cases}\sin n t, & 0<t<\frac{\pi}{2}, \\ \cos n t, & \frac{\pi}{2}<t<\pi .\end{cases}
$$

Let $J_{1}=\left(0, \frac{\pi}{2}\right), J_{2}=\left(\frac{\pi}{2}, \pi\right)$. It is obvious that $\left\{s_{n}\right\}$ are the eigen functions of the following spectral problem 
with a spectrum in boundary conditions

$$
\left\{\begin{array}{l}
u^{\prime \prime}(t)+\lambda^{2} u(t)=0, t \in J_{1} \cup J_{2}, \\
u(0)=u^{\prime}(\pi)=0 \\
u^{\prime}\left(\frac{\pi}{2}-0\right)=\lambda u\left(\frac{\pi}{2}+0\right),-\lambda u\left(\frac{\pi}{2}-0\right)=u^{\prime}\left(\frac{\pi}{2}+0\right) .
\end{array}\right.
$$

Concerning these issues see also the papers [11-14].

Another remarkable example is considered in V. A. Ilin's paper [15]. Here he considers a mixed problem with conjugation conditions at the inner point $x_{0} \in(0, l)$ with respect to the wave equation

$$
u_{t t}=a(x) u_{x x}, \quad x \in\left(0, x_{0}\right) \cup\left(x_{0}, l\right), t \in(0, T),
$$

with conditions

$$
\begin{gathered}
u(x, 0)=\varphi(x), \quad u_{t}(x, 0)=\psi(x), \\
u(0, t)=u(l, t)=0, u\left(x_{0}-0, t\right)=u\left(x_{0}+0, t\right), \\
a_{1}^{2} \rho_{1} u_{x}^{\prime}\left(x_{0}-0, t\right)=u_{2}^{2} \rho_{2} u_{x}^{\prime}\left(x_{0}+0, t\right),
\end{gathered}
$$

where

$$
a(x)= \begin{cases}a_{1}^{2}, & x \in\left(0, x_{0}\right), \\ a_{2}^{2}, & x \in\left(x_{0}, l\right),\end{cases}
$$

$a_{1}, a_{2}$ (wave velocity in medium) and $\rho_{1}, \rho_{2}$ (medium density) are positive constants, $a_{k}^{2} \rho_{k}$ are Young modules with additional condition of equality of passage time of wave the segments $\left[0, x_{0}\right]$ and $\left[x_{0}, l\right]: \frac{x_{0}}{a_{1}}=\frac{l-x_{0}}{a_{2}}$. The completeness in $L_{2}$ of the system of eigenfunctions of an ordinary differential operator that corresponds to this problem is established in the paper [16]. The close class of problems was earlier considered in the paper [17].

These examples very clearly demonstrate expediency of study of frame properties of the systems form (3). The present paper is devoted to investigation of frame property of system (3) in $L_{p} \equiv L_{p}(-\pi, \pi), 1<p<+\infty$. Previously some results of this paper were announced without proof in [18].

This work is structured as follows. In Section 2, we present needful information and facts from the theories of bases and close bases that will be used to obtain our main results. This section also contains the main assumptions about the functions $\alpha(t)$ and $\beta_{n}(t)$ which appear in formula (4). In Section 3, we state main results on the basicity of the perturbed system of exponents (3) in Lebesgue spaces $L_{p}, 1<p<+\infty$.

\section{Necessary Information and Main Assumptions}

In sequel we will need the following notion and facts from the theory of bases and frames. We will use the standard notation. $N$ will be the set of all positive integers; $\exists$ will mean "there exist(s)"; $\Rightarrow$ will mean "it follows"; $\Leftrightarrow$ will mean "if and only if"; $\exists$ ! will mean "there exists unique"; $K=R$ or $K=C$ will stand for the set of real or complex numbers, respectively; $\delta_{n k}$ is Kroneckers symbol, $\delta_{k}=\left\{\delta_{k n}\right\}_{k \in N}$. The Banach space will be called a $B$-space. $X^{*}$ is a space conjugate to space $X$. By $L[M]$ we denote the linear span of the set $M \subset X$, and $M$ will stand for the closure of $M$.

Definition 1. System $\left\{x_{n}\right\}_{n \in N} \subset X$ is said to be a basis for $X$ if $\forall x \in X, \exists !\left\{\lambda_{n}\right\}_{n \in N} \subset K: x=\sum_{n=1}^{\infty} \lambda_{n} x_{n}$.

Definition 2. System $\left\{x_{n}\right\}_{n \in N} \subset X$ is said to be complete in $X$ if $\overline{L\left[\left\{x_{n}\right\}_{n \in N}\right]}=X$. It is called minimal in $X$ if $x_{k} \notin \overline{L\left[\left\{x_{n}\right\}_{n \neq k}\right]}, \forall k \in N$.

Definition 3. System $\left\{x_{n}\right\}_{n \in N} \subset X$ is called $\omega$-linearly independent in $B$-space $X$, if from $\sum_{n=1}^{\infty} a_{n} x_{n}=0$ implies $a_{n}=0, \forall n \in N$.

It holds the following

Lemma 1. Let $X$ be a B-space with the basis $\left\{x_{n}\right\}_{n \in N}$ and $F: X \rightarrow X$ be a Fredholm operator. Then the following properties of the system $\left\{y_{n}=F x_{n}\right\}_{n \in N}$ in $X$ are equivalent:

1) $\left\{y_{n}\right\}_{n \in N}$ is complete;

2) $\left\{y_{n}\right\}_{n \in N}$ is minimal;

3) $\left\{y_{n}\right\}_{n \in N}$ is $\omega$-linearly independent;

4) $\left\{y_{n}\right\}_{n \in N}$ a basis isomorphic to $\left\{x_{n}\right\}_{n \in N}$.

We will need the following notions.

Definition 4. The systems $\left\{x_{n}\right\}_{n \in N}$ and $\left\{y_{n}\right\}_{n \in N}$ in a $B$-space $X$ with the norm $\|\cdot\|$ are said to be p-close, if $\sum_{n}\left\|x_{n}-y_{n}\right\|^{p}<+\infty$.

Definition 5. The minimal system $\left\{x_{n}\right\}_{n \in N} \subset X$ in a $B$-space $X$ with conjugated $\left\{x_{n}^{*}\right\}_{n \in N} \subset X^{*}$ is said to be a p-system if for $\forall x \in X:\left\{x_{n}^{*}(x)\right\}_{n \in N}^{n \in N} \in l_{p}$, where $l_{p}$ is an ordinary space of sequences $\left\{a_{n}\right\}_{n \in N}$ of scalars with the norm $\left\|\left\{a_{n}\right\}_{n \in N}\right\|_{l_{p}}=\left(\sum_{n}\left|a_{n}\right|^{p}\right)^{\frac{1}{p}}$.

In the case of basicity, such a system will be called a p-basis.

The following lemma is also valid.

Lemma 2. Let $X$ be a B-space with q-basis $\left\{x_{n}\right\}_{n \in N}$ and the system $\left\{y_{n}\right\}_{n \in N} \subset X$ be p-close to it: $\frac{1}{p}+\frac{1}{q}=1$, 
$1 \leq p<+\infty$. Then the expression $F x=\sum_{n} x_{n}^{*}(x) y_{n}$, generates a Fredholm operator in $X$, where $\left\{x_{n}^{*}\right\}_{n \in N} \subset X^{*}$ is a system conjugated to $\left\{x_{n}\right\}_{n \in N}$.

One can see these or other facts in the monographs $[3,19]$ and also in the papers [7,20-22]. We will need the following Krein-Milman-Rutman's Theorem [20].

Theorem KMR. $X$ be a B-space with the norm $\|\cdot\|$ and with the normed basis $\left\{x_{n}\right\}_{n \in N},\left\{x_{n}^{*}\right\}_{n \in N} \subset X^{*}$ be a system biorthogonal to it. If the system $\left\{y_{n}\right\}_{n \in N} \subset X$ satisfies the condition $\sum_{n=1}^{\infty}\left\|x_{n}-y_{n}\right\|<\eta^{-1}$, where $\eta=\sup _{n}\left\|x_{n}^{*}\right\|$, then it forms a basis isomorphic to $\left\{x_{n}\right\}_{n \in N}$ for $X$.

While obtaining the basic result, we will use the following easily provable lemma.

Lemma 3. Let $X$ be a B-space with the basis $\left\{x_{n}\right\}_{n \in N}$ and $\left\{x_{n}^{*}\right\}_{n \in N} \subset X^{*}$ be a system biorthogonal to $\left\{x_{n}\right\}_{n \in N}$. The system $\left\{y_{n}\right\}_{n \in N} \subset X$ differ from $\left\{x_{n}\right\}_{n \in N}$ by $a$ finitely many elements, i.e. $y_{n}=x_{n}, \forall n \geq n_{0}+1$. Then, if $\Delta_{n_{0}}=\operatorname{det}\left(x_{n}^{*}\left(y_{k}\right)\right)_{n, k=1, n_{0}}=0$ the system $\left\{y_{n}\right\}_{n \in N}$ is not minimal in $X$.

Proof. So, $X$ be a $B$-space with the basis $\left\{x_{n}\right\}_{n \in N}$ and $\left\{y_{n}\right\}_{n \in N} \subset X$ differ from $\left\{x_{n}\right\}_{n \in N}$ by finitely many elements, i.e. $y_{n}=x_{n}, \forall n \geq n_{0}+1$. Expand $y_{n}, n=\overline{1, n_{0}}$. by this basis.

$$
y_{k}=\sum_{n=1}^{n_{0}} a_{n k} x_{n}+y_{k, n_{0}}, k=\overline{1, n_{0}}
$$

where $y_{k, n_{0}}=\sum_{n=n_{0}+1}^{\infty} a_{n k} x_{n}$. Let $\Delta_{n_{0}}=0$. At first assume that $n_{0}=1$. Then, it is obvious that $\Delta_{n_{0}}=a_{11}=0$. As a result, it follows from expression (5) that $y_{1}$ belongs to the closure of the linear span $\left\{x_{n}\right\}_{n=n_{0}+1}^{\infty}$, and so the system $\left\{y_{n}\right\}_{n \in N}$ is not minimal. Consider the case $n_{0}=2$, i.e.

$$
\left.\begin{array}{l}
y_{1}=a_{11} x_{1}+a_{21} x_{2}+y_{1,2} \\
y_{2}=a_{12} x_{1}+a_{22} x_{2}+y_{2,2}
\end{array}\right\}
$$

where $\Delta_{2}=a_{11} a_{22}-a_{21} a_{12}=0$. It is obvious that if $\left|a_{1 k}\right|+$ $\left|a_{2 k}\right|=0$ for $k=1$ or $k=2$, then the system $\left\{y_{n}\right\}_{n \in N}$ is not minimal. Otherwise, excluding $x_{k}$ in (6), we have:

$$
\begin{gathered}
a_{12} y_{1}-a_{11} y_{2}=-\Delta_{2} x_{2}+a_{12} y_{1,2}-a_{11} y_{2,2} \\
=a_{12} y_{1,2}-a_{11} y_{2,2}, \\
a_{22} y_{1}-a_{21} y_{2}=a_{22} y_{1,2}-a_{21} y_{2,2} .
\end{gathered}
$$

It directly follows from these relations that $y_{1}\left(y_{2}\right)$ belongs to the closure of linear span of the remaining elements $\left\{y_{n}\right\}_{n \neq 1}\left(\left\{y_{n}\right\}_{n \neq 2}\right)$, i.e. $\left\{y_{n}\right\}_{n \in N}$ is not mini- mal in $X$. Consequently, for $\Delta_{2}=0$ the system $\left\{y_{n}\right\}_{n \in N}$ doesn't form a basis. This reasoning is taken to an arbitrary $n_{0} \in N$ very easily.

Before proceeding the main results, we accept the following basic assumptions concerning the functions of $\alpha(t)$ and $\beta_{n}(t)$.

1) $\alpha(t)$ is a piecewise-Holder function on $[-\pi, \pi]$, $\left\{t_{k}\right\}_{1}^{r}:-\pi=t_{0}<t_{1}<\cdots<t_{r}<t_{r+1}=\pi-$ are its discontinuity points of first kind;

Denote the jumps of the function $\alpha(t)$ at the points $\left\{t_{k}\right\}_{1}^{r}$ by $\left\{\alpha_{k}\right\}_{1}^{r}: \alpha_{k}=\alpha\left(t_{k}+0\right)-\alpha\left(t_{k}-0\right)$.

Let the condition

2) $\frac{\alpha_{k}}{\pi}-\frac{1}{p} \notin Z, \forall k=\overline{1, r}$, be fulfilled.

3) The functions $\beta_{n}(t)$ have the following asymptotic relations

$$
\beta_{n}(t)=O\left(\frac{1}{n^{\gamma}}\right), t \in(-\pi, \pi), \gamma \in(0,+\infty) .
$$

\section{Basic Results}

At first we consider the system of exponents

$$
\left\{\mathrm{e}^{\mathrm{i} \mu_{n}(t)}\right\}_{n \in Z},
$$

where $\mu_{n}(t)=n t-\alpha(t) \operatorname{sign} n, n \in Z$. For the basicity of system (8) in $L_{p}$, the results of the paper [23] will be used. Represent system (8) in the form

$$
\left\{\mathrm{e}^{-\mathrm{i} \alpha(t)} \mathrm{e}^{\mathrm{i} n t} ; \mathrm{e}^{\mathrm{i} \alpha(t)} \mathrm{e}^{-\mathrm{i}(n+1) t}\right\}_{n \in Z_{+}},
$$

( $Z_{+}$are non-negative integers). Let the condition 2) be fulfilled. Finding $\left\{n_{i}\right\}_{1}^{r} \subset Z$ from the following inequalities $\left(\frac{1}{p}+\frac{1}{q}=1\right)$ :

$$
-\frac{1}{q}<\frac{\alpha_{i}}{\pi}+n_{i-1}-n_{i}<\frac{1}{p}, i=\overline{1, r}, n_{0}=0,
$$

assume

$$
\omega=\frac{\alpha(-\pi)-\alpha(\pi)}{\pi}+n_{r} .
$$

Based on Theorem 1 of the paper [23] we can directly conclude the following

Statement 1. Let the conditions 1), 2) be fulfilled for the function $\alpha(t)$. Suppose that $\omega \neq \frac{1}{p}$. The system (9) forms a basis for $L_{p}, 1<p<+\infty$, (for $p=2$ a Riesz basis) if and only if it holds the inequality $-\frac{1}{q}<\omega<\frac{1}{p}$.

We will use the following statement obtaining from the results of the paper [24].

Statement 2. If system (9) forms a basis for $L_{p}$, 
$1<p<+\infty$, then it is isomorphic to the classic system of exponents $\left\{\mathrm{e}^{\mathrm{i} n t}\right\}_{n \in Z}$.

So, let system (8) form a basis for $L_{p}, 1<p<+\infty$. Denote by $\left\{\vartheta_{n}\right\}_{n \in Z} \subset L_{q}$ a system biorthogonal to it. Let $f \in L_{p}$ and $\left\{f_{n}\right\}_{n \in Z}$ be its biorthogonal coefficients by system (8), i.e. $f_{n}=\int_{-\pi}^{\pi} f(t) \overline{\vartheta_{n}(t)} \mathrm{d} t, n \in Z$, where (:) is complex conjugation. The following theorem can be directly concluded from Statement 2.

Theorem 1. Let system (8) forms a basis for $L_{p}$, $1<p<+\infty$. Then there hold:

1) Let $1<p \leq 2$ and $f \in L_{p}$. Then $\left\{f_{n}\right\}_{n \in Z} \in l_{q}$, and

$$
\left\|\left\{f_{n}\right\}_{n \in Z}\right\|_{l_{q}} \leq m_{p}\|f\|_{p},
$$

is fulfilled, where $m_{p}$ is a constant independent of $f,\|\cdot\|_{p}$ is an ordinary norm in $L_{p}$.

2) Let $p>2$ and the sequence of numbers $\left\{a_{n}\right\}_{n \in Z}$ belong to $l_{q}$. Then $\exists f \in L_{p}$ such that $f_{n}=a_{n}, \forall n \in Z$, moreover $\|f\|_{p} \leq M_{p}\left\|\left\{f_{n}\right\}_{n \in Z}\right\|_{l_{q}}$, where $M_{p}$ is a constant independent of $\left\{f_{n}\right\}_{n \in Z}$.

Now, study the basicity of system (3) in $L_{p}$. We have

$$
\begin{aligned}
\left|\mathrm{e}^{\mathrm{i} \lambda_{n}(t)}-\mathrm{e}^{\mathrm{i} \mu_{n}(t)}\right| & =\left|\mathrm{e}^{\mathrm{i} \beta_{n}(t)}-1\right|=\left|\sum_{k=1}^{\infty} \frac{\beta_{n}^{k}(t)}{k !}\right| \\
& \leq \sum_{k=1}^{\infty} \frac{\left(M n^{-\gamma}\right)^{k}}{k !}=c n^{-\gamma},
\end{aligned}
$$

where $c$ is a constant independent of $n$. The last inequality follows from (7).

Consider the different cases.

1) Let $1<p \leq 2, \gamma>\frac{1}{p}$. We have

$$
\sum_{n}\left\|\mathrm{e}^{\mathrm{i} \lambda_{n}(t)}-\mathrm{e}^{\mathrm{i} \mu_{n}(t)}\right\|_{p}^{p} \leq c_{p} \sum_{n} \frac{1}{|n|^{\gamma p}}<+\infty .
$$

Assume that all the conditions of Statement 1 are fulfilled. Then, system (8) forms a basis for $L_{p}$. Thus, by Statement 2 it forms a $q$-basis for $L_{p}$ in this case. Let $\left\{\vartheta_{n}\right\}_{n \in Z}$ be a system biorthogonal to it. Consider the operator $F: L_{p} \rightarrow L_{p}$ :

$$
F f=\sum_{n} \vartheta_{n}(f) \mathrm{e}^{\mathrm{i} \lambda_{n}(t)}, f \in L_{p},
$$

where $\vartheta_{n}(f)=\int_{-\pi}^{\pi} f(t) \overline{\vartheta_{n}(t)} \mathrm{d} t, n \in Z$. By Lemma 2 operator (12) is Fredholm in $L_{p}$. It is easy to see that $F\left[\mathrm{e}^{\mathrm{i} \mu_{n}(t)}\right]=\mathrm{e}^{\mathrm{i} \lambda_{n}(t)}, \forall n \in Z$. Then, the statement of Lemma 1 is valid for system (3).

2) Let $2<p<+\infty, \gamma>\frac{1}{q}$. It is clear that $q<p$.
Consequently, for $\forall f \in L_{p}$ it is valid $\|f\|_{q} \leq c_{p}\|f\|_{p}$, where $c_{p}$ depends only on $p$. Assume that all the conditions of Statement 1 are fulfilled. Consequently, system (8) forms a basis for $L_{p}$. It is clear that $f \in L_{q}$ and $1<q<2$. Then, from Theorem 1 we obtain that $\left\{f_{n}\right\}_{n \in Z} \in l_{p}$, where $\left\{f_{n}\right\}_{n \in Z}$ are the orthogonal coefficients of $f$ by system (8). From the same theorem we obtain:

$$
\left\|\left\{f_{n}\right\}_{n \in Z}\right\|_{l_{p}} \leq m_{q}\|f\|_{q} \leq M_{p}\|f\|_{p}, \forall f \in L_{p},
$$

where the constant $M_{p}$ is independent of $f$. Thus, system (8) forms a $p$-basis in $L_{p}$. It is easy to see that systems (3) and (8) $q$-close in $L_{p}$. Consider operator (12). Further, we behave similarly to case I. Hence the validity of the following theorem is proved.

Theorem 2. Let asymptotic Formula (4) hold, the function $\alpha(t)$ satisfy the conditions 1),2) and for the function $\beta_{n}(t)$ the relations (7) be valid. Assume that it holds

$$
-\frac{1}{q}<\omega<\frac{1}{p}, \gamma>\max \left\{\frac{1}{p} ; \frac{1}{q}\right\},
$$

where $\gamma=\min _{k} \gamma_{k}, \omega$ is defined from expressions (10), (11). Then, the following properties for system (3) in $L_{p}$ are equivalent:

1) Complete;

2) Minimal;

3) $\omega$-linearly independent;

4) Forms a basis isomorphic to $\left\{\mathrm{e}^{\mathrm{int}}\right\}_{n \in Z}$.

In sequel, we will consider a case, when $\gamma>1$. In this case, it is obvious that it holds $\sum_{n=-\infty}^{\infty}\left\|\mathrm{e}^{\mathrm{i} \lambda_{n}(t)}-\mathrm{e}^{\mathrm{i} \mu_{n}(t)}\right\|_{p}<+\infty$. Let all the conditions of Theorem 2 be fulfilled. Then the system $\left\{\mathrm{e}^{\mathrm{i} \mu_{n}(t)}\right\}_{n \in Z}$ forms a basis for $L_{p}$. Denote by $\left\{\vartheta_{n}\right\}_{n \in Z} \subset L_{q}$ a system biorthogonal to it. Assume $\vartheta=\sup _{n}\left\|\vartheta_{n}\right\|_{q}$. It is clear that

$$
\exists n_{0} \in N: \sum_{|n| \geq n_{0}+1}\left\|\mathrm{e}^{\mathrm{i} \lambda_{n}(t)}-\mathrm{e}^{\mathrm{i} \mu_{n}(t)}\right\|_{p}<\vartheta^{-1}
$$

Consider the functions

$$
\tilde{\lambda}_{n}(t)= \begin{cases}\lambda_{n}(t), & |n|>n_{0}, \\ \mu_{n}(t), & |n| \leq n_{0} .\end{cases}
$$

Thus, it holds

$$
\sum_{n=-\infty}^{\infty}\left\|\mathrm{e}^{\mathrm{i} \tilde{\lambda}_{n}(t)}-\mathrm{e}^{\mathrm{i} \mu_{n}(t)}\right\|_{p}<\vartheta^{-1} .
$$

Then, as it follows from Theorem KMR, the system $\left\{\mathrm{e}^{\mathrm{i} \tilde{\pi}_{n}(t)}\right\}_{n \in Z}$ forms a basis isomorphic to $\left\{\mathrm{e}^{\mathrm{i} \mu_{n}(t)}\right\}_{n \in Z}$ for 
$L_{p}$. System (3) and the basis $\left\{\mathrm{e}^{\mathrm{i} \tilde{z}_{n}(t)}\right\}_{n \in Z}$ differ by a finitely many elements. By $\left\{\tilde{\vartheta}_{n}\right\}_{n \in Z}$ denote a biorthogonal system to this basis. Consider

$$
\mathrm{e}^{\mathrm{i} \lambda_{k}(t)}=\sum_{|n| \leq n_{0}} a_{n k} \mathrm{e}^{\mathrm{i} \mu_{n}(t)}+\sum_{|n|>n_{0}} a_{n k} \mathrm{e}^{\mathrm{i} \lambda_{n}(t)}, \forall k:|k| \leq n_{0},
$$

It is obvious that $a_{n k}=\tilde{\vartheta}_{n}\left(\mathrm{e}^{\mathrm{i} \lambda_{k}(t)}\right)=\int_{-\pi}^{\pi} \mathrm{e}^{\mathrm{i} \lambda_{k}(t)} \overline{\tilde{\vartheta}_{n}(t)} \mathrm{d} t, n$, $k \in Z$. Denote by $\Delta_{n_{0}}$ the following determinant

$$
\Delta_{n_{0}}=\operatorname{det}\left(a_{i j}\right)_{i, j=-n_{0}, n_{0}} .
$$

It is clear that if $\Delta_{n_{0}} \neq 0$, in the expansion (13) the elements $\mathrm{e}^{\mathrm{i} \mu_{k}(t)}, k=\overline{-n_{0}, n_{0}}$ may be replaced by the elements $\mathrm{e}^{\mathrm{i} \lambda_{k}(t)}, k=\overline{-n_{0}, n_{0}}$. Then the system $\left\{\mathrm{e}^{\mathrm{i} \tilde{z}_{n}(t)}\right\}_{n \in Z}$ forms a basis for $L_{p}$, since $\forall f \in L_{p}$ has the expansion $f=\sum_{n=-\infty}^{\infty} \tilde{\vartheta}_{n}(f) \mathrm{e}^{\mathrm{i} \tilde{z}_{n}(t)}$. Hence, it directly follows that if $\Delta_{n_{0}} \neq 0$, then $\forall f \in L_{p}$ has an expansion by system (3), i.e. it is complete in $L_{p}$. Consider the operator

$$
\begin{aligned}
& \tilde{F} f=\sum_{n=-\infty}^{\infty} \tilde{\vartheta}_{n}(f) \mathrm{e}^{\mathrm{i} \lambda_{n}(t)} . \text { We have } \\
& \tilde{F} f=\sum_{n=-\infty}^{\infty} \tilde{\vartheta}_{n}(f) \mathrm{e}^{\mathrm{i} \tilde{\lambda}_{n}(t)}+\sum_{n=-\infty}^{\infty} \tilde{\vartheta}_{n}(f)\left[\mathrm{e}^{\mathrm{i} \lambda_{n}(t)}-\mathrm{e}^{\mathrm{i} \tilde{z}_{n}(t)}\right] \\
& =f+\sum_{n=-n_{0}}^{n_{0}} \tilde{\vartheta}_{n}(f)\left[\mathrm{e}^{\mathrm{i} \lambda_{n}(t)}-\mathrm{e}^{\mathrm{i} \tilde{\lambda}_{n}(t)}\right]=(I+T) f,
\end{aligned}
$$

where $I: L_{p} \rightarrow L_{p}$ is an identity operator, and $T$ is an operator generated by the second summand. Fredholm property $F$ in $L_{p}$ follows from finite-dimensionality of the operator $T$. It is clear that

$$
\tilde{F}\left[\mathrm{e}^{\mathrm{i} \tilde{\lambda}_{n}}(t)\right]=\mathrm{e}^{\mathrm{i} \lambda_{n}(t)}, \forall n \in Z .
$$

Then from Lemma 1 we obtain the basicity of system (3) in $L_{p}$. Conversely, if system (3) forms a basis for $L_{p}$, then as it follows from Lemma $3, \Delta_{n_{0}} \neq 0$. Thus, we established that under accepted conditions system (3) forms a basis for $L_{p}$ if the determinant determined by expression (14) is not zero.

Thus, we proved the following.

Theorem 3. Let all the conditions of Theorem 2, where $\gamma>1$, be fulfilled. The determinant $\Delta_{n_{0}}$ is determined by expression (14). System (3) forms a basis for $L_{p}$, $1<p<+\infty$, if and only if $\Delta_{n_{0}} \neq 0$.

Now, consider the case when $\omega \notin\left(-\frac{1}{q}, \frac{1}{p}\right)$. Let for example, $\frac{1}{p}<\omega<\frac{1}{p}+1$. In this case, as it follows from
Theorem 1 of the paper [23], the system

$$
\left\{\mathrm{e}^{\mathrm{i} \alpha(t)}\right\} \cup\left\{\mathrm{e}^{\mathrm{i} \mu_{n}(t)}\right\}_{n \in Z},
$$

forms a basis for $L_{p}$. Consider the system

$$
\{e(t)\} \cup\left\{\mathrm{e}^{\mathrm{i} \lambda_{n}(t)}\right\}_{n \in Z},
$$

where $e \in L_{p}$ is a function. Let the conditions 1),2) be fulfilled for system (3) and $\gamma>\max \left\{\frac{1}{p} ; \frac{1}{q}\right\}$. Then, it is easy to see that system (16) and basis (15) are $\tilde{p}$-close in $L_{p}$, where $\tilde{p}$ is determined by the formula

$$
\tilde{p}= \begin{cases}p, & 1<p \leq 2, \\ q, & p>2\end{cases}
$$

Consequently, system (3) is not complete in $L_{p}$. The remaining cases, when $\omega>\frac{1}{p}$, are proved in the similar way.

Consider a case, when $\omega<-\frac{1}{q}$, for example,

$\omega \in\left(-\frac{1}{q}-1,-\frac{1}{q}\right)$. In this case, again as it follows from Theorem 1 of the paper [23], the system

$$
\left\{\mathrm{e}^{\mathrm{i} \mu_{n}(t)}\right\}_{n \neq 0},
$$

forms a basis for $L_{p}$. If the conditions 1), 2) are fulfilled, then basis (17) and the system $\left\{\mathrm{e}^{\mathrm{i} \lambda_{n}(t)}\right\}_{n \neq 0}$ are $\tilde{p}$-close in $L_{p}$. Consequently, system (3) is not minimal in $L_{p}$. The remaining cases, when $\omega<-\frac{1}{q}$, are proved similarly. Therefore, we obtain the following final result for the basicity of system (3) in $L_{p}$.

Theorem 4. Let asymptotic formula (4) hold, where the functions $\alpha(t)$ and $\beta_{n}(t)$ satisfy the conditions 1$)$, $2), 3)$. The variable $\omega$ be determined from relations (10), (11) and let $\gamma>\max \left\{\frac{1}{p} ; \frac{1}{q}\right\}$. Then for $\omega<-\frac{1}{q}$ system (3) is not minimal in $L_{p}$; for $\omega>\frac{1}{p}$ it is not complete in $L_{p}$. For $-\frac{1}{q}<\omega<\frac{1}{p}$ the following properties of system (3) in $L_{p}$ are equivalent:
1) Complete;
2) Minimal;
3) $\omega$-linearly independent;
4) Forms a basis isomorphic to $\left\{\mathrm{e}^{\mathrm{int}}\right\}_{n \in Z}$;
5) $\Delta_{n_{0}} \neq 0$, where $\Delta_{n_{0}}$ is determined by expression 
(14).

Indeed, equivalence of properties 1)-4) follows directly from Lemma 1. Equivalence of conditions 4) and 5) is proved.

\section{Conclusions}

Taking into account the obtained results, we can summarize this work as follows.

Perturbed system of exponents, the phase of which may has different asymptotic behavior in different parts of the basic interval $(-\pi, \pi)$, is studied in this work. It should be noted that it's probably the first time the problem of basicity is considered for such a system. Under certain conditions on the functions defining the phase, we prove that this system may have a finite defect in $L_{p}$, $1<p<+\infty$. Moreover, it either forms a basis for $L_{p}$, or it is not complete and not minimal in $L_{p}$.

\section{Acknowledgements}

The authors express their deepest gratitude to Professor B. T. Bilalov, for his attention and valuable guidance to this article.

\section{REFERENCES}

[1] R. Paley and N. Wiener, "Fourier Transforms in the Complex Domain," American Mathematical Society, Providence, 1934.

[2] N. Levinson, "Gap and Density Theorems," American Mathematical Society, Providence, 1940.

[3] R. M. Young, "An Introduction to Non-Harmonic Fourier Series," Springer, Berlin, 1980, p. 246.

[4] A. M. Sedletskii, "Classes of Analytic Fourier Transformations and Exponential Approximations," Fizmatlit, Moscow, 2005.

[5] Ch. Heil, “A Basis Theory Primer,” Springer, Berlin, 2011, p. 534. doi:10.1007/978-0-8176-4687-5

[6] O. Christensen, "An Introduction to Frames and Riesz bases," Springer, Berlin, 2003, p. 440.

[7] D. L. Russell, "On Exponential Bases for the Sobolev Spaces Over an Interval," Journal of Mathematical Analysis and Applications, Vol. 87, No. 2, 1982, pp. 528-550. doi:10.1016/0022-247X(82)90142-1

[8] X. He and H. Volkmer, "Riesz Bases of Solutions of Sturm-Lioville Equations," Journal of Fourier Analysis and Applications, Vol. 7, No. 3, 2001, pp. 297-307. doi:10.1007/BF02511815

[9] H. Miklos, "Inverse Spectral Problems and Closed Exponential Systems," Annals of Mathematics, Vol. 162, No. 2, 2005, pp. 885-918. doi:10.4007/annals.2005.162.885

[10] A. M. Sedletskii, "Nonharmonic Analysis," Functional
Analysis, Itogi Nauki i Tekhniki Seremennaya Matematika i ee Prilozheniya Tematicheskie Obzory, Vol. 96, 2006, pp. 106-211.

[11] L. H. Larsen, "Internal Waves Incident upon a Knife Edge Barrier," Deep Sea Research, Vol. 16, No. 5, 1969 , pp. 411-419.

[12] S. A. Gabov and P. A. Krutitskii, "On Larsen's Nonstationary Problem," Zhurnal Vychislitel'noi Matematiki i Matematicheskoi Fiziki, Vol. 27, No. 8, 1987, pp. 11841194.

[13] P. A. Krutitskii, "Small Non-Stationary Vibrations of Vertical Plates in a Channel with a Stratified Fluid," USSR Computational Mathematics and Mathematical Physics, Vol. 28, No. 6, 1988, pp. 166-176.

[14] E. I. Moiseev and N. Abbasi, "Basis Property of Eigenfunctions of the Generalized Gasedynamic Problem of Frankl with a Nonlocal Oddness Condition and with the Discontinuity of the Gradient of Solution," Differential Equations, Vol. 45, No. 10, 2009, pp. 1452-1456.

[15] V. A. Ilin, "Mixed Problem Describing the Damping Process of a Bar Consisting of Two Sections of Different Density and Elasticity Provided that the Time of Wave's Passage in Each of These Sections Coincide," Trudi Instituta Matematiki i Mekhaniki Uro RAN, Vol. 269, 2010, pp. 132-141.

[16] I. S. Lomov, "Non-Smooth Eigenfunctions in Problems of Mathematical Physics," Differential Equations, Vol. 47, No. 3, 2011, pp. 358-365.

[17] L. M. Lujina, "Regularity of Spectral Problems with Additional Conditions at the Inner Points," Matematicheskie Zametki, Vol. 49, No. 3, 1991, pp. 151-153.

[18] B. T. Bilalov and S. M. Farahani, "On Perturbed Bases of Exponential Functions with Complex Coefficients," Transactions of NAS of Azerbaijan, Vol. 56, No. 4, 2011, pp. 45-50.

[19] I. Singer, "Bases in Banach Spaces, I," Springer, Berlin, 1970, p. 673. doi:10.1007/978-3-642-51633-7

[20] I. T. Hochberg and A. S. Markus, "On Stability of Bases of Banach and Hilbert Spaces," Izvestiya Akademii Nauk Moldavskoj SSR, No. 5, 1962, pp. 17-35.

[21] B. T. Bilalov and T. R. Muradov, "On Equivalent Bases in Banach Spaces," Ukrainian Mathematical Journal, Vol. 59, No. 4, 2007, pp. 551-554. doi:10.1007/s11253-007-0040-1

[22] B. T. Bilalov, "Bases from Exponents, Cosines and Sines Being Eigen Functions of Differential Operators," Differential Equations, Vol. 39, No. 5, 2003, pp. 1-5.

[23] B. T. Bilalov, "Basicity of Some Systems of Exponents, Cosines and Sines," Differential Equations, Vol. 20, No. 1, 1990, pp. 10-16.

[24] B. T. Bilalov, "On Isomorphism of Two Bases," Fundamentalnaya i Prikladnaya Matematika, Vol. 1, No. 4, 1995, pp. 1091-1094. 